Solid State Communications, Vol.31, pp.35-38.

Pergamon Press Ltd. 1979. Printed in Great Britain.

\title{
CYCLOTRON RESONANCE IN AN InAS-GaSb SUPERLATTICE
}

\author{
H. Bluyssen, J.C. Maan, P. Wyder \\ Research Institute for Materials, University of Nijmegen, \\ Toernooiveld, Nijmegen, The Netherlands \\ L.L. Chang and L. Esaki \\ IBM Thomas J. Watson Research Center, \\ Yorktown Heights, New York 10598, U.S.A.
}

(Received 29 March 1979 by A.R. Miedema)

\begin{abstract}
Optical transmission in the far infrared region in an InAs-GaSb guperlattice is studied as a function of magnetic field and frequency. Cyclotron resonance is observed for electrons confined in the InAs conduction band whose ground state is shifted to higher enersy due to the periodic superlattice potential. These measurements confirm the calculated quantisation of the energy levels in a superlattice by ascertaining the effective mass directly.
\end{abstract}

A superlattice is a structure consisting of ultrathin, periodic layers of two different semiconductors (layer thickness in the order of $100 \mathrm{~A}$ which is about 20 lattice constants per layer). This artificially introduced one-dimensional periodic potential leads to a strong modification of the original band structure of the host materials in the superlattice direction. The original Brillouin zone is divided into subzones with subzone boundaries at wavevector values corresponding to the superlattice periodicity. The superlattice as a whole can be considered as a completely new, highly anisotropic material whose electronic and optical properties are determined not only by the band parameters of the basis material, but also by the superlattice periodicity, which can be varied at wil1 1,2 . Most of the work up to now has been restricted to the relatively simple structures of GaAs-Ga $1-\mathrm{Al}^{\mathrm{As}}{ }^{1-3}$. Recently a new class of superlattices consisting of consecutive layers of InAs and GaSb has been considered ${ }^{4}$. Shubnikor-de Hass measurements 5 on this type of superlattice show clearly the extreme anisotropy of the superlattice system, and good agreement is found between the measured and the colculated Fermi energy. However, the agreement between the calculated effective mass and the effective mass determined indirectly from the temperature dependence of the Shubnikov-de Hass oscillations was not satisfactory for all samples.

In this paper we report the first observation of cyclotron resonance in a superlattice, and therefore the first direct and accurate determination of the effective mass. The superlattice under investigation was that of InAs and $\mathrm{GaSb}$. This structure is especially interesting because due to the strong energy dependence of the effective mass, its determination provides direct information about the energy levels in the superlattice.

The periodic arrangement of two semiconductors in a superlattice gives rise to a one- dimensional periodic potential. Therefore the problem of calculatins the band structure strongly resembles the well known Kronig- Penney problem for free electrons in a rectangular periodic potential. However, in the case of a superlattice, Bloch wave functions ghould be used instead of free electron wave functions. Taking into account this fact and matching the wave functions of different materials and their derivatives at the successive boundaries of the superlattice potential taken to be along the $z$-direction, one gets 4 $\cos \left(\mathrm{kd}_{0}\right)=\cos \left(\mathrm{k}_{1} \mathrm{~d}_{1}\right) \cos \left(\mathrm{k}_{2} \mathrm{a}_{2}\right)-$

$\frac{1}{2}\left(\frac{i k_{1}+i_{1} / u_{1}}{i k_{2}+u_{2} / u_{2}}+\frac{i k_{2}+i_{2} / u_{2}}{i k_{1}+u_{1} / u_{1}}\right) \sin \left(k_{1} a_{1}\right) \sin \left(k_{2} a_{2}\right)$

Here, the subscripts 1 and 2 refer to the two different materials, $k_{1}, k_{2}$ are the original $k_{2}$ wave vectors of the host materials, $k$ is the wave vector of the superlattice, $d$ is the superlatice periodicity, $d_{1}, d_{2}$ are the layer thicknesses of the host materibls, and $u_{1}, u_{2}$ are the cell-periodic parts of the Bloch function at an arbitrary boundary. Eq. (1) implies the well known fact that not all oxiginal $k_{2}$ values of the host materials are allowed anymore by the superlattice periodicity. More specifically, the lowest allowed $k_{2}$ value is non-zero. Knowing the dispersion relation of the basis materials, the energy bands can be calculated once the allowed $k_{2}$ values are known. Since $k_{i}=0$ is not allowed anymore, this leads to a shift in energy of the band edges in a superlattice. For an InAs-Gasb superlattice the lowest conduction subband and the highest valence subband calculated in this way results from matching at the boundaries of the light hole Gasb valence band wave function to the light electron InAs conduction band wave function. Since we are interested in electrons 
only we do not consider the heavy hole valence band which does not couple to the other two bands. The heavy hole bands in a superlattice have to be considered separately 4 . The Fermi energy can be obtained by integration of the density of states of each subband, knowing the total electron concentration $n$. This calculated Fermi energy can be verifieg by means of the Shubnikov-de Haas effect 5,6 .

Table I summarizes the relevant characteristics of the investigated sample. The carrier concentration and the mobility were derived from Hall measurements. The subband energies and width of the two lowest conduction bands and the Fermi energy were calculated as described above. The meterial parameters used for this calculation are $0.42 \mathrm{eV}$ and $0.81 \mathrm{eV}$ for the energy gaps, band edge masses $\mathrm{m} / \mathrm{m}$ of electrons of 0.023 and 0.048 , and for the light holes of 0.025 and 0.052 for InAs and GaSb respectively, while the bottom of the InAs conduction band is taken to be $0.1 \mathrm{eV}$ below the top of the GaSb valence band7. The Fermi energy is calculated as $\mathrm{E}_{\mathrm{F}}=39 \mathrm{meV}$ obove the lowest subband edge, which implies that only this band is occupied. This band originates from the InAs conduction band which is shifted to higher lattice potential are in the z-direction, Eq. (2) is modified in the following way: The bottom of the band in a superlattice is given by Eq. (2) for $k_{x}=0, k=0$, and $k_{\text {, has the }}$ lowest value allowed by the superlatfice periodicity. The energy corresponding to this level is $E_{1}$, which is just the shift in the InAs conduction band. In a magnetic field, the remaining quantum numbers $k_{x}$ and $k_{y}$ are replaced by $\left(N+\frac{1}{2}\right) \frac{h e B}{m_{0}^{*}}$; here $N$ is the Landau level number. Combining with $\mathrm{Eq}$. (2) one obtains the energy levels of a superlattice in a magnetic field

$$
\left(\mathrm{N}+\frac{1}{2}\right) \frac{\mathrm{heB}_{\mathrm{eB}}}{\mathrm{m}_{\mathrm{O}}}=\mathrm{E}_{\mathrm{N}}\left(1+\frac{\mathrm{E}_{\mathrm{II}}}{\mathrm{E}_{\mathrm{G}}}\right)-\mathrm{E}_{1}\left(1+\frac{\mathrm{E}_{1}}{\mathrm{E}_{\mathrm{G}}}\right) \text {, }
$$

and an effective mass

$$
m^{*}\left(E_{1}, B\right)=m_{0}^{*}\left(1+\frac{E_{E+1}\left(E_{1}, B\right)+E_{g}\left(E_{1}, B\right)}{E_{G}}\right)
$$

Where $\mathrm{m}^{*}$ is again the effective mass at the bottom $8 f$ the conduction band of the bulk InAs; $\mathrm{E}_{1}\left(\mathrm{E}_{1}, \mathrm{~B}\right)$ can be calculated from $\mathrm{Bq}$. (3). Therefôre, a determination of the effective mass provides a direct means to verify the calcula-

Table I

Superlattice sample characteristics.

InAs layer thickness

Gasb layer thickness

number of periods

carrier concentration (Hall effect)

carrier mobility (Hall effect)

first subband energy (subband width)(calculated)

second subband energy (subband width)(calculated)

calculated Fermi energy above first subband

measured Fermi energy above first subband

(Shubnikov-de has effect, using $\mathrm{m}^{\mathbf{3}}=0.043 \mathrm{~m}$ )

$\begin{array}{ll}\mathrm{d}_{1} & 65 \AA \\ \mathrm{d}_{2} & 80 \AA \\ \mathrm{n} & 125 \\ \mathrm{n}_{\mathrm{e}} & 4.810^{17} \mathrm{~cm}^{-3} \\ \mu & 7300 \mathrm{~cm}^{-2} / \mathrm{vs} \\ \mathrm{E}_{1}\left(\Delta \mathrm{E}_{1}\right) & 186(10) \mathrm{meV} \\ \mathrm{E}_{2}\left(\Delta \mathrm{E}_{2}\right) & 465(6) \mathrm{meV} \\ \mathrm{E}_{F_{\text {calc }}} & 39 \mathrm{meV} \\ \mathrm{E}_{\mathrm{F}_{\text {meas }}} & 40 \mathrm{meV}\end{array}$

energies by an anount $\mathrm{F}_{1}$. This implies that in a cyclotron resonance experiment the observed mass has to be related to the InAs conduction band mass. All other masses occur at energies relatively far away from the Fermi level. We now focus on this band to calculate the energy levels of the superlattice in a magnetic field. In $a$ two band model, using the k.p method, the approximate expression of the dispersion relation for the InAs conduction band valid over the energy range of the present experiments, is given by 8

$\frac{h^{2} k_{x}^{2}}{2 m_{0}^{n}}+\frac{h^{2} k_{y}^{2}}{2 m_{0}^{n}}+\frac{h^{2} k_{z}^{2}}{2 m_{0}^{n}}=E\left(1+\frac{E}{E_{G}}\right)$

where $E$ is the energy measured with respect to the InAs conduction band edge, and $E_{\text {, and }}$ ar are the InAs band gap and the band edge effective mass. In a superlattice and in a magnetic field where both magnetic rield and super- ted shift $E_{1}$ in the InAs conduction band. For this purpose ve measured the effective mass by measuring the change in the Far Infrared Radiation (FIR) tranamission due to cyclotron resonance as a function of frequency and magnetic field.

The FIR tranamission experiments vere done at three different wavelengths $(\lambda=118.8 \mathrm{\mu m}$, $\lambda=70.6 \mu \mathrm{m}$ and $\lambda=57 \mathrm{\mu m}$ ) using an optically pumped FIR laser system. The magnetic fields were senerated by one of the Bitter coils of the Hijmegen High Magnetic Fields Installation. The transmitted radiation was detected by a He-cooled Si-bolcmeter. The tranmission signal was normalized by means of a reference signal to eliminate rluctuation of the laser output power.

Fig. 1 shows the observed change in transmission for the three different wavelengths invest gated. A clearly resolred though fairly broad resonance is observed. The inset in Fig. 1 shows the peak positions vs FIR energy and mag- 


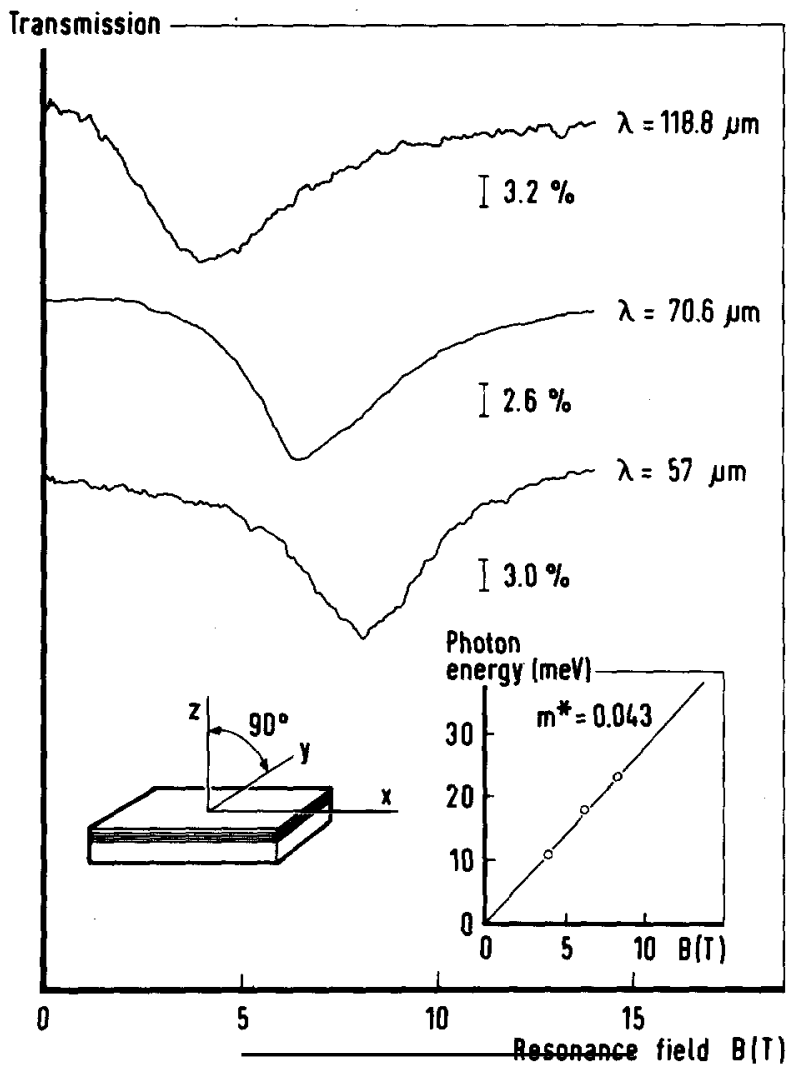

Fig. 1 : Transmission signal for an InAs-GaSb superlattice as a function of magnetic field for different frequencies. The field is parallel to the direction of the super lattice potential. The inset shows the transmission minima as a function of the magnetic field and photon energy. The drawn line represents the fit of an effective mass of $0.043 \mathrm{~m}$ to the data.

netic field. From the slope of the observed linear dependence, an effective mass of $0.043 \mathrm{~m}_{0}$ is derived. Using this effective mass a Fermi energy can be derived from the periodicity of the observed Shubnikov-de Haas oscillations. This results in Fermi energy of $40 \mathrm{meV}$ which agrees very well with the calculated value of $39 \mathrm{meV}$. For bulk InAs the energy dependence of the effective mass is given by $m^{x}(E)=m_{0}^{x}\left(1+\frac{2 E}{x_{0}}\right)$. Using this equation the observed effectife mass would occur at an enerBY of $182 \mathrm{meV}$ whereas for the superlattice sample under investigation the Fermi energy is only $40 \mathrm{meV}$. The observed effective mass there- fore directly demonstrates the lifting of the bulk conduction band due to the superlattice periodicity.

In order to identify the relevant cyclotron resonance transitions, we show in Fig. 2 the calculated splitting of the subbands as a function of magnetic field using Eq. (3). Transitions occur between conduction band states below and above the Fermi level, and the observed transitions are indicated in the figure. The effective masses at the three resonant fields can be calculated by using Eq. (4), and can be compared with the observed values (Table II). Considering that the calculation contains no adjustable parameters (only commonIy accepted literature values for the bulk material are used) and that spinsplitting and energy dependence of the 8 -factor' are completely neglected, the agreement between measured and calculated masses is quite satisfactory.

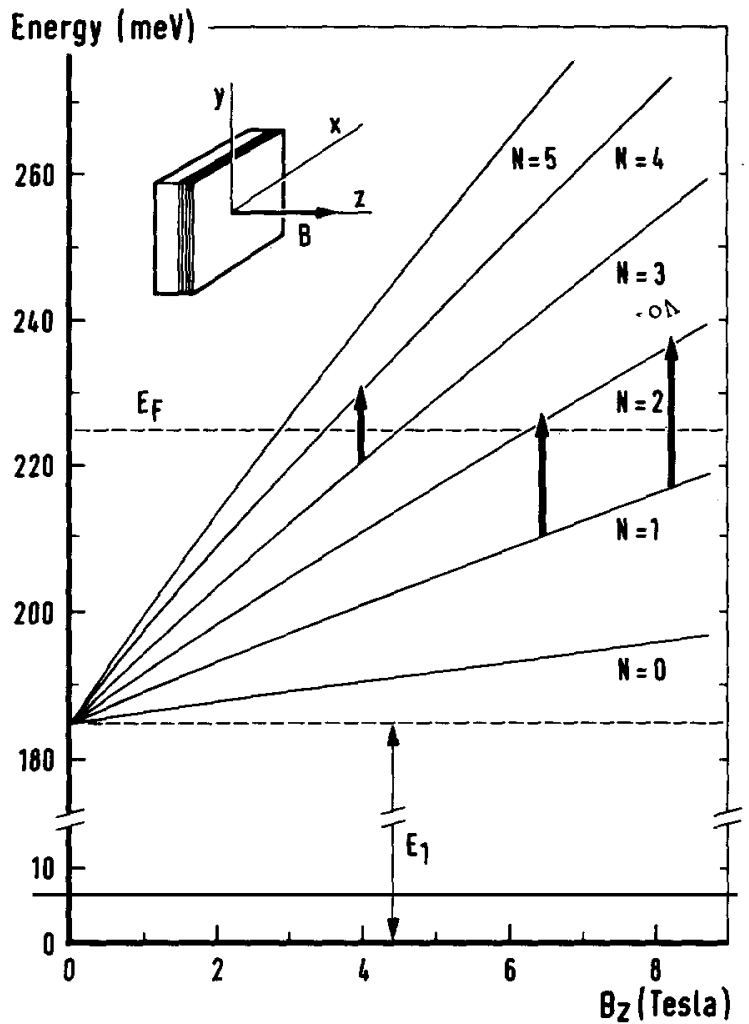

Fig. 2 : Calculated energy levels of an InAs-GaSb superlattice in a magnetic field. The vertical solid lines represent the measured cyclotron resonance transition. For the energy scale, the bulk InAs conduction band edge is taken as the zero level.

Table II

Comparison between measured and calculated effective masses.

$\begin{array}{ccc}\text { Resonance Field } & \mathbf{m}^{\mathbf{*}} \text { calculated } & \mathbf{m}^{\mathbf{*}} \text { measured } \\ \mathrm{B}(\mathrm{Tesla}) & & \\ 3.95 & 0.0476 & 0.044 \pm 0.001 \\ 6.39 & 0.0468 & 0.042 \pm 0.001 \\ 8.16 & 0.0480 & 0.044 \pm 0.001\end{array}$


It should be noted that both the calculated and observed masses are somewhat lower for $6.39 \mathrm{~T}$ than for the other two fields. The reason for this may lie in the relative positions of the Fermi enersy with respect to the Lendau levels: The transition at this field occurs at a somewhat lower energy than those at the other two fields, as can be seen from Fig. 2.

In conclusion it may be stated that for the firgt time cyclotron resonance in a superlattice has been observed. The measured effective mass can only be explained by taking into account the shift in the energy band brought about by the quantisation caused by the superlattice periodicity. This observation therefore is a direct demonstration of the quantisation of the energy bands in a superlattice.

Acknowledgement - Part of this work performed at the University of Nijmegen has been supported by the "Stichting voor Fundamenteel Onderzoek der Materie" (FOM) with financial support of the "Nederlandse Organisatie voor Zuiver Wetenschappelijk Onderzoek" (ZWO). Work at the IBM Thomas $J$. Watson Research Center was partially supported under an Army Research office Contract.

\section{REFERENCES}

1. ESAKI, L. and TSU, R., IBM J. Res. Develop. 14, 61 (1970).

2. ESAKI, L. and CHANG, L.L, Thin Solid Films $\overline{36}, 285$ (1976).

3. DINGLE, R., "Confined Carrier Quantum States in Ultrathin Semiconductor Heterostructures" in: Festkörperprobleme XV, 21 (1975).

4. SAI HALASZ, G.A., TSU, R, and ESAKI, L., Appl. Phys. Lett. 30, 651 (1977).

5. SAKAKI, H., CHANG, L.L., SAI HALASZ, G.A., CHANG, C.A.and ESAKI, L., Solid State Commun. 26. $589(1978)$.

6. CHANG, L.L., SAKAKI, H., CHANG, C.A. and ESAKI, L., Phys. Rev. Lett. 38, 1489 ( 1977 ).

7. Handbook of Electronic Materials, Vol. II, III-V Semiconducting Compounds, ed. M. Neuberger (IFI/Plenum, New York, 1971 ).

8. KANE, E.0., J. Phys. Chem. Solids 12, 181 (1959).

9. PIDGEON, C.R., MITCHELL, D.L. and BROWN, R.N., Phys. Rev. 154, $737(1967)$. 